\title{
Modelling Tactical Driving Manoeuvres with GA-INTACT
}

\author{
H. Tawfik ${ }^{1}$ and P. Liatsis ${ }^{2}$ \\ ${ }^{1}$ School of Computing, Liverpool Hope University, Liverpool, UK \\ tawfikh@hope.ac.uk \\ ${ }^{2}$ School of Engineering and Mathematical Sciences, City University, UK \\ p.liatsis@city.ac.uk
}

\begin{abstract}
This work concerns the design and development of a driving simulation system, which exhibits intelligent driving behaviour at the tactical level, as part of a traffic simulation environment. Our tactical driving system using genetic algorithms, named GA-INTACT, accounts for the subject vehicle and other vehicles positions and speed parameters in the surrounding traffic condition, and selects favourable speed change and lane transition actions for the 'subject' vehicle, according to safety, speed and driving behaviour criteria. Simulation results demonstrated that the adoption of the Genetic Algorithms approach for obtaining near-optimum driving solutions eliminates the need for learning driving patterns, and allows the efficient handling of the complex nature of tactical driving modelling problem. The role of the driving behaviour in influencing the outcome of the driver's decision is emphasised, an aspect that was not treated sufficiently in previous tactical driving simulation approaches.
\end{abstract}

\section{Introduction}

The development of Autonomous Vehicle Navigation (AVN) systems remains an active research area for the automotive industry, and several AVN systems $[3,13]$ have been tested in structured environments, such as motorways. A key issue to the success of any AVN system is the systematic performance evaluation of the underlying sensing algorithms [5] in a variety of traffic scenes and driving scenarios.

The Road Simulation Environment (ROSE) [12] has been developed as a traffic and driving simulation platform for testing obstacle detection, lane detection and driving modelling systems. This has been achieved through the construction of a visual database of traffic scenes; and the generation of geometrically valid 3-D roads [7], and driving scenarios by simulating driving decision-making procedures.

We focus here on the tactical driving part which contributes to limited research in the modelling of this complex driving activity. Our tactical driving system, GAINTACT, selects favourable tactical driving actions for the 'subject' vehicle according to safety, speed and driving behaviour criteria, using a genetic algorithm.

\section{Related Tactical Driving Simulation Research}

Considerable progress was made in automating various strategic and operational driving aspects, compared to limited success in developing intelligent tactical driving 
models [4]. Typical applications of tactical modelling targeted automatic cars and robots driving in simulated environments and adopted various rule-based, neural networks and evolutionary techniques. The main challenges associated with rule-based approaches [4,10] are the difficulty of modifying existing rules and implementing new ones, and poor results in unanticipated driving situations. Typical problems that arise with neural networks solutions [1,9] include a biased behaviour towards a particular driving style, exhibiting similar mistakes to those in the training data, and the lack of a criterion for the determination of satisfactory a representation for tactical driving in the training set. Other research attempts used genetic algorithms (GAs) to model tactical driving tasks $[2,11]$ by exploiting their power in solving complex problems without the need for a comprehensive understanding of tactical driving as for the rule-based case or for a representative training set as with neural networks.

\section{INtelligent TACTical Driving System Based on Genetic Algorithms (GA-INTACT)}

The objective of the driving simulation module of ROSE is to provide driving scenarios where vehicles drive at desirable speeds, maintain safety gaps, and closely resemble real driving, in terms of speed changes and lane transitions.

A 'Subject driver' centred approach was adopted such that driving journey refers to the changing driving situation in the immediate vicinity of the subject vehicle driver. The subject vehicle driving actions in terms of lane and speed changes are determined by GA-INTACT. A dual carriageway road type with two lanes on each traffic direction (Fig.1) was chosen for this application. It permits a variety of manoeuvres to be performed, and is genaralisable to other road structures such as Motorways.



Fig. 1. Layout of the dual carriageway

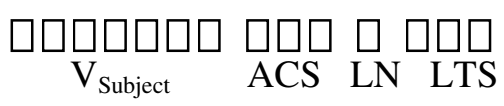

Fig. 2. GA solution representation

A number of application specific assumptions were made, as follows:

- Only the subject vehicle has the ability to perform intelligent tactical manoeuvres. The driving scenarios of other vehicles are predetermined and prior information with regard to each vehicle's position and speed, at any stage, is also available.

- A flowing traffic without interruptions, roundabouts or junctions, is assumed.

- A five-second interval (FSI) is the updating period for the tactical driving steps as it allows adequate time to perform realistic speed changes and lane transitions [12].

- The two-second minimum gap: This safety distance is the distance required to allow for a two second time gap to the vehicle ahead, at the subject vehicle speed, such that: 


$$
\mathrm{D}_{\text {two_sec }}=\mathrm{V} \times 2
$$

where $\mathrm{D}_{\text {two_sec }}$ is the two-sec distance in metres, and $\mathrm{V}$ is the vehicle speed in $(\mathrm{m} / \mathrm{s})$.

- Driver Behaviour Model (DBM): The DBM input takes the parameters of the driver's age, gender, experience, and personality, and returns a 0 to 1 output, corresponding to a range of very risky to very cautious driving behaviours, as follows:

$$
\mathrm{DBM}=\mathrm{w}_{1} \mathrm{DBM}_{\mathrm{A}}+\mathrm{w}_{2} \mathrm{DBM}_{\mathrm{E}}+\mathrm{w}_{3} \mathrm{DBM}_{\mathrm{P} / \mathrm{G}}
$$

where $\mathrm{DBM}_{\mathrm{A}}, \mathrm{DBM}_{\mathrm{E}}$, and $\mathrm{DBM}_{\mathrm{P} / \mathrm{G}}$ are the different $\mathrm{DBM}$ contributions resulting from the driver's age, experience and personality/gender factors, respectively [12].

\subsection{Genetic Alogorithms}

A genetic algorithm search was adopted for the selection of optimum tactical manoeuvres for the subject vehicle as it can provide a powerful means of obtaining optimum solutions for complex problems and eliminate the need for obtaining training data. GAs are stochastic search methods that adopt mechanisms of natural selection and genetics for carrying out their optimisation tasks [6].

The GA solution in GA-INTACT is a tactical decision in the form of a change in speed, acceleration rate, and lane and the type of lane transition, which is then optimised with respect to tactical driving criteria. We use a 14-bit binary string that encodes the speed, acceleration, lane parameters (Fig. 2), as follows:

- $\quad V_{\text {Subject }}$ is the target speed of the subject vehicle at the end of the FSI. The 7 bits allow for a 1-kph resolution of a 0 to $128 \mathrm{kph}$ speed range.

- ACS (Acceleration Scale): this scaling factor corresponds to the change in acceleration/deceleration over the FSI [8]. A 3-bit field allows for scenarios such as (000) for a 'very sharp' acceleration rate and (111) for a 'very smooth' deceleration rate.

- $\quad$ LN is the subject vehicle's lane number at the end of the FSI where LN $=0$ is for the slow lane and $\mathrm{LN}=1$ is for the fast lane.

- LTS (Lane Transition Scale): this reflects the manner by which the lane change is performed in terms of smoothness/sharpness. LTS ranges from 'very smooth' fast-to-slow lane transition (LTS $=7 / 7$ ) to 'very sharp' slow-to-fast transition $(\mathrm{LTS}=0 / 7)$.

\subsection{GA Fitness Function Design}

Four main design optimisation criteria were defined, namely safety, speed, driving behaviour, and 'keeping to the slow lane'. The overall GA fitness function can be described as the weighted sum of the 4 evaluation measures, as follows:

$$
\text { GA fittness }=\mathrm{w}_{1} \cdot \mathrm{f}_{\text {Safety }}+\mathrm{w}_{2} \cdot \mathrm{f}_{\text {Speed }}+\mathrm{w}_{3} \cdot \mathrm{f}_{\text {Behaviour }}+\mathrm{w}_{4} \cdot \mathrm{f}_{\text {Slow }}
$$

Three conditions were imposed to reject unrealistic or unlawful driving solutions at every FSI maneouvre, based on min. gap and max. speed and acceleration limits: 
$\mathrm{G}(.) \geq \mathrm{G}_{\min }$ (.) : Gap to the vehicle ahead which is below minimum safety gap.

$\mathrm{V}(.) \geq \mathrm{V}_{\text {Opt }}$ (.) : Subject vehicle drives faster than its optimum speed $\mathrm{V}_{\text {Opt }}$.

$\mathrm{a}(.) \geq \mathrm{a}_{\text {Max }}($.$) : Vehicle exceeds its max. acceleration/deceleration rate \mathrm{a}_{\max }[8]$.

\subsubsection{Safety Fitness ( $\left(_{\text {Safety }}\right)$}

The objective of the safety function is to drive the subject vehicle such that the gap to the vehicle ahead is reduced, while maintaining the minimum safety gap, as follows:

$$
\mathrm{f}_{\text {Safety }}=\frac{\mathrm{S}_{\text {Safety }}}{\mathrm{S}_{\text {Safety }}+\left(\mathrm{G}(\mathrm{t}+\mathrm{T})-\mathrm{G}_{\text {min }}(\mathrm{t}+\mathrm{T})\right)} .
$$

where: T = FSI (i.e., 5 seconds),

$\mathrm{G}(\mathrm{t}+\mathrm{T})$ is the actual gap ahead of the subject vehicle at the end of the manoeuvre.

$\mathrm{G}_{\min }(t+T)$ is the minimum gap ahead of the subject vehicle at time $(t+T)$.

The $\mathrm{G}$ gap is the distance from the subject vehicle to the vehicle ahead.

$\mathrm{G}_{\min }$ is based on the two-second rule, with the addition of the DBM related term [12]. $S_{\text {Safety }}$ is a scaling parameter based on the initial Gap $G(t)$ is and minimum gap $G_{\min }(t)$.

\subsubsection{Speed Fitness $\left(\mathbf{f}_{\text {Speed }}\right)$}

The $f_{\text {Speed }}$ function encourages solutions according to which the subject vehicle drives at speeds near or equal to the optimum speed, as follows:

$$
\mathrm{f}_{\text {Speed }}=\frac{\mathrm{S}_{\text {Speed }}}{\mathrm{S}_{\text {Speed }}+\left(\mathrm{V}_{\text {Opt }}(\mathrm{t}+\mathrm{T})-\mathrm{V}_{\text {Subject }}(\mathrm{t}+\mathrm{T})\right)}
$$

The optimum speed $V_{\text {Opt }}$ is dependent on the road design speed $V_{D}($.$) , the vehicle's ac-$ celeration capabilities, and its driver behaviour DBM [12].

$\mathrm{V}_{\text {Subject }}(\mathrm{t}+\mathrm{T})$ is the subject vehicle speed at the end of the FSI.

$\mathrm{V}_{\text {Opt }}(\mathrm{t}+\mathrm{T})$ is the optimum subject vehicle speed at the end of the FSI

$S_{\text {Speed }}$ is the $f_{\text {Speed }}$ function scaling parameter based on $V_{\text {Subject }}(t)$ is the initial subject vehicle speed and $\mathrm{V}_{\mathrm{Opt}}(\mathrm{t})$ is the initial optimum subject vehicle speed.

\subsubsection{Driving Behaviour Fitness ( $f_{\text {Behaviour }}$ )}

The $\mathrm{f}_{\text {Behaviour }}$ gives reflects the compatibility of the subject vehicle manoeuvre with the driving behaviour (DBM). It encourages tactical actions in the form of LTS, ASC, and SCS (Speed Change Scale) [12], which are coherent with DBM and discourages actions that are inconsistent with it. $\mathrm{f}_{\text {Behaviour }}$ can be written as:

$$
\mathrm{f}_{\text {Behaviour }}=\mathrm{f}(\text { LTS, ACS, SCS, DBM })
$$

DBM is the subject driver behaviour model output value, LTS is the lane transition scale, ACS is the acceleration rate scale, and SCS is the speed change scale [12].

A heuristic model was formulated with data points that define the correspondence between the DBM, LTS, SCS and ACS input terms and the output value of $f_{\text {Behaviour }}$ [12]. According to this model, when DBM=0.9 (overcautious drivers), high performance 
scenarios $\left(\mathrm{f}_{\text {Behaviour }}>0.8\right)$ involve low acceleration rates, decreasing speeds (SCS $\backslash$ ACS $>0.6$ ), and smooth lane transitions from fast to slow lanes (LTS $>0.7$ ). On the other hand, very low fitness values are produced, when a 'cautious' driver carries out high acceleration and speed rates and sharp lane transition manoeuvres.

\subsection{4 'Keeping to Slow Lane' Fitness ( $\mathbf{f}_{\text {Slow lane }}$ )}

This fitness term comes to noticeable effect, when there are 'almost' equal gaps ahead of the subject vehicle to the immediate vehicles on both slow and fast lanes, with these vehicles driving at similar speeds. The influence of $\mathrm{f}_{\text {Slow lane }}$ is such that, when a similar traffic condition are present at both lanes, the subject vehicle would end up on the slow lane. The $\mathrm{f}_{\text {Slow Lane }}$ function was formulated as follows:

$$
\mathrm{f}_{\text {Slow lane }}=\left(\mathrm{LN}_{\max }-\mathrm{LN}\right) \times\left(\frac{\mathrm{S}_{\text {Slow lane }}}{\mathrm{S}_{\text {Slow lane }}+\left|\mathrm{V}_{\text {Slow }}-\mathrm{V}_{\text {Fast }}\right|+\left|\mathrm{G}_{\text {Slow }}-\mathrm{G}_{\text {Fast }}\right|}\right)
$$

where $\mathrm{V}_{\text {Slow }}$ and $\mathrm{V}_{\text {Fast }}$ are the speeds of the vehicles ahead, on slow and fast lanes.

$\mathrm{G}_{\text {Slow }}$ and $\mathrm{G}_{\text {Fast }}$ are the gaps of the vehicles ahead on the slow and fast lanes, respectively. If there are no vehicles ahead, then $\mathrm{G}_{\text {Slow/Fast }}=200 \mathrm{~m}$, and $\mathrm{V}_{\text {Slow } / \text { Fast }}=128 \mathrm{kph}$.

$\mathrm{LN}$ is the lane number of the subject vehicle at the end of the FSI.

$\mathrm{LN}_{\max }$ the maximum number of lanes per traffic direction number.

The $\mathrm{S}_{\text {Slow lane }}$ scaling parameter adjusts the sharpness of $\mathrm{f}_{\text {Slow lane }}$ based on DBM [12].

\section{Simulation Results}

This section presents examples of tactical driving results produced by GA-INTACT. A 3 FSI traffic journey length was selected to evaluate the performance of the system.

A graphical representation of the behaviour of the subject vehicle's actions is presented with plots of the key GA optimisation criteria as follows:

Subject vehicle speed $\left(\mathrm{V}_{\text {Subject }}\right)$ and optimum speed $\left(\mathrm{V}_{\text {Opt }}\right)$ Vs (FSI) or (T);

The minimum gap $\left(\mathrm{G}_{\mathrm{min}}\right)$ and actual gap to the vehicle ahead $(\mathrm{G})$ Vs time steps $(\mathrm{T})$.

Lane number (LN), LTS and ACS values at each time step (T).

The speed and distance values are approximated to the nearest kph for speed calculations, and nearest meter for gap calculations, while LTS and ACS values are approximated to the nearest decimal. An initial LTS and ACS value of 0.5 is assumed.

Due to the dynamic nature of the driving conditions and constraints, tactical manoeuvres are assessed in comparison to the optimum speed and minimum safety gaps.

Our traffic scenario has the following conditions: Design speed=120 kph, Subject vehicle starting lane $\mathrm{LN}=0$, Starting speed $=80 \mathrm{kph}$, speed of the first vehicle ahead on the slow lane $=90 \mathrm{kph}$, and speed of the first vehicle ahead on the fast lane $=100 \mathrm{kph}$.

In order to observe the effect of the driving behaviour on the tactical manoeuvres, the same traffic conditions were simulated with three DBM values: DBM=0.9 'overcautious' driver, $\mathrm{DBM}=0.5$ 'standard' and $\mathrm{DBM}=0.1$ 'risky' driver.

We use the U.K road layout standards in which the slow lane refers to the left lane while the fast lane refers to the right lane. This layout structure and subsequent calculations can be changed in the simulation to meet other road layout conventions. 
Case 1: overcautious driver $(\mathrm{DBM}=0.9)$, Figs $3 \& 4$.

$1^{\text {st }}$ manoeuvre (Time step 0 to 1 ): the subject vehicle accelerates from $80 \mathrm{kph}$ to 102 $\mathrm{kph}$ to match its optimum speed $\left(\mathrm{V}_{\mathrm{Opt}}\right)$, thus reducing the gap ahead from $60 \mathrm{~m}$ to $58 \mathrm{~m}$, in a 'smooth' manner (ACS=0.4), on the same lane.

$2^{\text {nd }}$ manoeuvre (Time step 1 to 2 ): the gap ahead of the subject vehicle is reduced to the optimum gap from $58 \mathrm{~m}$ to $50 \mathrm{~m}$ on the same lane, by decelerating smoothly (ACS $=0.7$ ) to a speed of $92 \mathrm{kph}$, a speed level near that of the vehicle ahead.

$3^{\text {rd }}$ manoeuvre (Time step 2 to 3 ): The optimum gap of $48 \mathrm{~m}$ is matched, by readjusting the subject vehicle's speed to that of the vehicle ahead on the left lane. The speed is reduced smoothly (ACS=0.7) from $92 \mathrm{kph}$ to $90 \mathrm{kph}$.

Case 2: standard driver $(\mathrm{DBM}=0.5),[12]$

$1^{\text {st }}$ manoeuvre: the subject vehicle accelerates from $80 \mathrm{kph}$ to match its optimum speed of $104 \mathrm{kph}$, thus reducing the gap ahead from $60 \mathrm{~m}$ to $57 \mathrm{~m}$, close to the optimum gap. The subject vehicle increases speed in a 'smooth' manner (ACS=0.4).
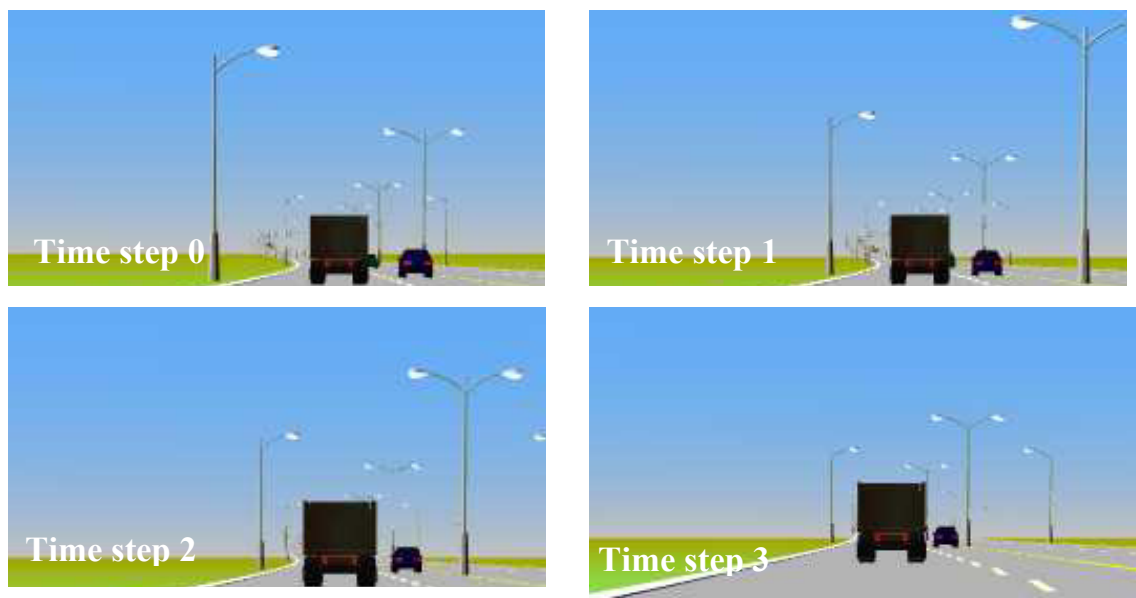

Fig. 3. Three 5-second step journey from the subject vehicle's perspective for case 1
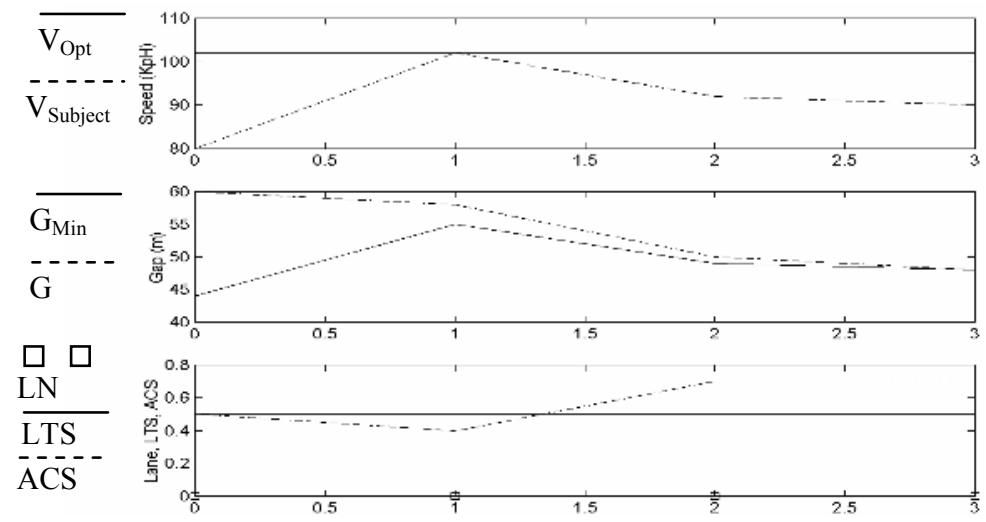

Fig. 4. Five-second time step changes of the subject vehicle's behaviour, for case 1 
$2^{\text {nd }}$ manoeuvre: the gap ahead of the subject vehicle is decreased to $52 \mathrm{~m}$ and is therefore kept near the optimum gap of $50 \mathrm{~m}$. This is achieved by decelerating smoothly (ACS $=0.6)$ to a speed of $91 \mathrm{kph}$, and making no lane change.

$3^{\text {rd }}$ manoeuvre: The subject vehicle carries out a smooth lane transition $(\mathrm{LTS}=0.4$ ) from the slow to the fast lane $(\mathrm{LN}=1)$. The speed is increased from $91 \mathrm{kph}$ to the optimum of $104 \mathrm{kph}$ on the fast lane, in a smooth manner (ACS=0.4). The gap to the 'new' fast-lane vehicle ahead, which had been traveling at $00 \mathrm{Kph}$, is now very large.

Case 3: risky driver $(\mathrm{DBM}=0.1)$, [12]

$1^{\text {st }}$ manoeuvre: the subject vehicle accelerates from $80 \mathrm{kph}$ to $107 \mathrm{kph}$, a speed close to its optimum $111 \mathrm{kph}$. This speed allows a 56m gap to the vehicle ahead which is a near-optimum gap length, as the minimum gap is $55 \mathrm{~m}$. The vehicle also stays on the slow lane, and carries out the speed increase in a 'very sharp' manner (ACS $=0.1$ ).

$2^{\text {nd }}$ manoeuvre: The subject vehicle carries out a very sharp lane change (LTS $=0.1$ ) from the slow to the fast lane $(\mathrm{LN}=1)$. This allows it to increase its speed from 107 $\mathrm{kph}$ to the optimum $111 \mathrm{kph} \mathrm{s}$. A sharp acceleration type was carried out $(\mathrm{ACS}=0.3$ ).

$3^{\text {rd }}$ manoeuvre: no speed or lane change, reducing the gap is reduced from 90 to $80 \mathrm{~m}$.

Results show that GA-INTACT aims at driving at maximum possible speeds and maintaining minimum gaps. When there exists a wide range of optimisable speed and safety gap combinations, high DBM 'cautious' drivers, have the tendency of travelling on the slow lane and optimising the safety distance. 'Standard' drivers produce a driving action that follows the general need to optimise the speed and the safety distance, changing lanes when necessary. 'Risky' drivers increase their speed and travel on the fast lane. In addition, varrying DBMs result in different approaches to speed and lane changes. Cautious drivers perform smooth, acceleration and lane transitions, while risky drivers perform these manoeuvres in a sharp manner.

Optimisation of the safety and speed criteria can be thought of as driving at the maximum possible speed, while keeping the safety distance to the other vehicles.

The use of DBM represents a distinct property of the GA approach in our GAINTACT system, and allows the generation of more realistic and broader types of tactical manoeuvres, and the possibility to 'steer' the subject vehicle's driving journey.

The current 14-bit GA solution size means that the problem under consideration has a relatively small search space. The GA efficiency would be more apparent when using GA-INTACT for more complicated configurations. For more than 2 lanes per direction, the chromosome size needs to be increased such that it allows enough resolution to accommodate for extra LTS patterns. In a more sophisticated traffic scenario in which other vehicle are equipped with GA-INTACT, the solution size can increase significantly to accommodate for interactions between vehicles driving in the vicinity of each other. This leads to a larger search space to explore and would require more parallel optmisation of the tactical manoeuvres of all interacting vehicles.

\section{Conclusions}

The GA-INTACT system adopts a genetic search approach to generate highperformance tactical driving solutions with regards to safety, speed and driving behaviour criteria. The driver behaviour element was particularly emphasised during the 
tactical driving design. GA-INTACT performs a variety of speed and lane change actions at various traffic situations, and generates tactical manoeuvres which optimise driving safety, efficiency and the driver's behaviour-manoeuvre compatibility criteria.

The work may be extended to a more versatile driving simulation system, which accommodates for driving decisions that relate to issues such as approaching junctions, traffic signals, and taking suitable exits. A more sophisticated traffic layout would be that where other vehicles, in particular those in the subject vehicle's vicinity are themselves equipped with GA-INTACT systems carry out speed and lane changes in a highly dynamic manner, an remains as a future research challenge.

\section{References}

1. Arain, M.A.,et al.: Action planning for the collision avoiding system using neural networks. Proc Intelligent Vehicles Sym. (1993) 119-124

2. Baluja, S., and Sukthankar, R.: Prototyping intelligent vehicle modules using evolutionary algorithms. Evolutionary Algorithms in Engineering Applications. Springer-Verlag (1998)

3. Campell, N.W.,et al.: Autonomous road vehicle navigation. Engineering Applications of Artificial Intelligence. Vol.7(2), (1990) 177-190.

4. Ehlet, P.A.M., and Rothkrantz, L.J.M.: Microscopic traffic simulation with reactive driving agents. Proc. $4^{\text {th }}$ IEEE Intelligent Transportation Systems Conf. USA (2001) 860-865

5. Foresti, G., et al.: A distributed approach to 3D scene recognition. IEEE Trans. Vehicular Technology. vol.43(2), (1994) 389-406

6. Goldberg, D.E.: Genetic algorithms in search, optimisation, and machine learning. Addison-Wesley (1989)

7. Liatsis, P., and Tawfik, H..M.: Two dimensional road shape optimisation using genetic algorithms. Mathematics and Computers in simulation. Vol.5, (1999) 19-31

8. Leutzbach, W.: Introduction to the theory of traffic flow. Splinger-verlag, Berlin (1988)

9. Lyons, G., and Hunt, J.: Traffic models - a role for neural networks. Proc. of neural networks and combinatorial traffic models. (1993) 71-79

10. Reece, D.A., and Shafer, S.A.: A computational model of driving for autonomous vehicles. Transportation research. vol.27A(1), (1993) 23-50

11. Sukthankar, R., Baluja, S., and Hancock, J.: Multiple adaptive agents for tactical driving. International Journal of Artificial Intelligence. Vol. 9(1), (1998)

12. Tawfik, H.M.: A graphical simulation environment for modelling of road and traffic scenarios. PhD Thesis. Control Systems Centre, UMIST, UK (2000)

13. Tribe, R.T., et al.: Intelligent driver support. 2nd World Congress on Intelligent Transport Systems. Yokohama, Japan (1995) 